\title{
Improved polymer solar cell performance by engineering of cathode interface
}

\author{
J.K. Baral ${ }^{1,2,3, a}$, R. Izquierdo ${ }^{2, b}$, M. Packirisamy ${ }^{3}$, and V.-V. Truong ${ }^{1}$ \\ ${ }^{1}$ Department of Physics, Concordia University, Montréal, Québec, Canada \\ ${ }^{2}$ NanoQAM, Département d'informatique, Université du Québec à Montréal (UQAM), Montréal, Québec, Canada \\ ${ }^{3}$ Optical-Bio Microsystems Laboratory, Mechanical \& Industrial Engineering Department, Concordia University, \\ Montréal, Québec, Canada
}

Received: 3 February 2011 / Accepted: 30 May 2011

Published online: 18 August 2011 - (c) EDP Sciences 2011

\begin{abstract}
By engineering the interface between the intermediate photoactive layer and the cathode aluminum $(\mathrm{Al})$ electrode, through the introduction of ultra-thin layers of various materials, in a standard bulk heterojunction (BHJ) polymer solar cell (PSC) fabricated of regioregular poly(3-hexylthiophene) (rr-P3HT) and phenyl-C61-butyric acid methyl ester (PCBM), the power conversion efficiency (PCE) has been effectively improved. The devices fabricated using individual single interlayer of bathocuproine (BCP), lithium fluoride (LiF) and Buckminster fullerene $\mathrm{C}_{60}$ have shown optimal efficiencies of $\sim 2.40 \%, \sim 3.21 \%$ and $\sim 1.92 \%$ respectively. Further improvement of the photovoltaic efficiency was achieved by introducing a composite bilayer made of $\mathrm{LiF}$ in combination with $\mathrm{BCP}$ as well as with $\mathrm{C}_{60}$ at the $\mathrm{BHJ} /$ cathode interface. The best results were obtained by interposing a $9 \mathrm{~nm}$ of $\mathrm{C}_{60}$ interlayer in combination with a $0.9 \mathrm{~nm}$ thick LiF layer, with the PCE of the PV cells being effectively increased up to $3.94 \%$ which represents an improvement of $23 \%$ as compared to the standard device with LiF interlayer alone. The photocurrent density $\left(J_{s c}\right)$ versus voltage $(V)$ characteristic curves shows that the increase of the efficiency is essentially due to an increase in $J_{s c}$. Moreover, all the sets of devices fabricated using various interlayers over a certain range of thickness exhibit an optimum PCE that is inversely proportional to the series resistance of the $\mathrm{PV}$ cells. We presume that interposing a $\mathrm{C}_{60} / \mathrm{LiF}$ layer at the interface could repair the poor contact at the electron acceptor/cathode interface and improve the charge career extraction from the BHJ.
\end{abstract}

\section{Introduction}

The rapidly growing demand for the global green energy in association with the increase in carbon dioxide $\left(\mathrm{CO}_{2}\right)$ emission attributed to the combustion of fossil fuels are the main driving forces for the development of inexpensive renewable, sustainable, clean and green energy sources [1]. Organic photovoltaic (OPV) devices have drawn considerable amount of attention in research and development due to their easy solution processable fabrication, low cost effectiveness, mechanical flexibility, eco-friendly, bio-degradable and the general applicability of organic materials [2,3]. Typically, an OPV cell is made up of a light-absorbing and charge career generating layer which is sandwiched between two different metal electrodes. One of the electrodes should be transparent and is usually made of indium-tin-oxide (ITO), and the other one is reflective and aluminum (Al) is often used [4]. The most used material in polymer solar cells for photo-absorbing

\footnotetext{
a e-mail: jbar@alcor.concordia.ca
}

b e-mail: izquierdo.ricardo@uqam.ca and charge generation layer is a nanoblend of regioregular poly(3-hexylthiophene) (rr-P3HT) and phenyl- $\mathrm{C}_{61}$ butyric acid methyl ester (PCBM) as donor and acceptor, respectively [5]. The working principle of a bulk heterojunction (BHJ) solar cell consists of a few steps, such as photo-exciton generation, exciton diffusion, exciton dissociation into charge carriers, charge transport and charge collection [6]. Upon absorption of a photon, an electron is excited from the ground state to its excited state, leaving a hole with opposite electric charge, to which it is attracted by Coulombic force, forming an electron-hole (e-h) pair, also called exciton. It is a well known fact that organic materials have relatively low dielectric constant $(\varepsilon) \sim 3-4$ and exciton has a relatively high binding energy $\sim 0.2-0.4 \mathrm{eV}$ $[6,7]$. Hence, it is impossible to split the exciton pairs into free charge carriers only by thermal energy at room temperature. This is why an efficient electron acceptor is used to dissociate the strongly bound excitons into free charge carriers. However, as the diffusion length is only $\sim 3-10 \mathrm{~nm}$ in conjugated systems, the excitons have to separate into free carriers within this length scale [6]. 
Therefore, excitons have to diffuse to the nearest donor and acceptor interface before they recombine and decay. After separation, charges are eventually collected and extracted at the opposite electrodes to produce a photocurrent in the external circuit [4].

In OPVs, it is a well known fact that introduction of a conventional buffer layer of poly(3,4-ethylenedioxythiophene) doped with poly(styrenesulfonate) (PEDOT:PSS) layer between the ITO anode electrode and the active layer improves the hole transport which results in an improvement of the fill factor (FF) and PCE significantly. For further improvement of polymer solar cell performances, the research and development on the interfacial modification between the BHJ active layer and the cathode electrode is an ongoing topic of discussion in recent days [8-13]. Various types of materials have been used as interfacial layers in the field of organic solar cells and each type of layer could have a different role [8]. Several reports suggested that interface engineering could be done upon inserting a thin buffer layer of fullerene- $\mathrm{C}_{60}$ or $\mathrm{BCP}$ or $\mathrm{LiF}$ at the $\mathrm{BHJ} /$ cathode interface in order to improve the performances of organic electronic devices [14-21]. The main effects of such layers are: (i) to prevent the unwanted charge recombination of the photogenerated charge carriers by keeping them away from the electrodes, (ii) to modify the interface to achieve a sharp and homogeneously ordered interface, (iii) to tune the effective work function of the electrodes, in order to make the interfacial zone suitable and favorable for excellent carrier extraction [21]. In this paper, we try to make a systematic study of the influence of thickness and the nature of various materials, as interlayer at the active layer/cathode interface, on the performance of polymer solar cells.

\section{Fabrication and experimental details}

In this work, the polymer photovoltaic cells were fabricated by spin-coating a nanoblend of P3HT:PCBM in a 1:0.8 wt/wt ratio, sandwiched between two parallel metallic electrodes, one is transparent and is made of indiumtin-oxide (ITO) of high work function, whereas the other one is reflective and made of aluminum $(\mathrm{Al})$ of relatively low work function. The ITO electrode and the Al electrode act as anode and cathode, respectively. The glass pre-coated with ITO was first patterned by lithography and etching according to the desired geometric configuration. The patterned ITO glass substrates were thoroughly cleaned by immersion in ultrasonic bath of acetone, isopropyl alcohol (IPA) and de-ionized water $\left(\mathrm{DI}-\mathrm{H}_{2} \mathrm{O}\right)$ sequentially for $20 \mathrm{~min}$ each. The patterned and cleaned ITO was covered by spin-coating with a $30 \mathrm{~nm}$ thick layer of poly(3,4-ethylenedioxythiophene) doped with highly conducting electronic grade poly(styrenesulfonate) (PEDOT:PSS), which was used as received from H.C. Starck (CLEVIOS ${ }^{\mathrm{Tm}} \mathrm{P}$ VP Al 4083). The glass/ITO/ PEDOT:PSS substrates were baked at $120{ }^{\circ} \mathrm{C}$ for $1 \mathrm{~h}$ in the general ambient air before being transferred to a glove box (nitrogen-filled mBRAUN) for the photoactive layer deposition. The regioregular poly(3-hexylthiophene)
(rr-P3HT) and phenyl-C 61 -butyric acid methyl ester (PCBM) were used as received from Rieke and SigmaAldrich, respectively. P3HT and PCBM were separately dissolved at a ratio of $10 \mathrm{mg} / \mathrm{ml}$ and $8 \mathrm{mg} / \mathrm{ml}$ with 1,2-dichlorobenzene (DCB), respectively. The two solutions were then stirred at $40{ }^{\circ} \mathrm{C}$ for $\sim 1 \mathrm{~h}$ inside the glove box. By mixing together the two parent solutions at a 1:0.8 wt/wt ratio, a P3HT:PCBM solution was prepared for the photoactive layer of the BHJ cell. Prior to the spincoating, the P3HT:PCBM solution was stirred at $40{ }^{\circ} \mathrm{C}$ for $\sim 16 \mathrm{~h}$ inside the glove box. The final blend was spuncoat at $1000 \mathrm{rpm}$ for $60 \mathrm{~s}$ then transferred to a petri dish and left to dry for about $1 \mathrm{~h}$. The spun-coat P3HT:PCBM film was measured to be $\sim 160 \mathrm{~nm}$ thick. The sample was then baked in the glove box at $140{ }^{\circ} \mathrm{C}$ for $1 \mathrm{~h}$. The samples were removed from the glove box and exposed to air then introduced in the evaporator for thermal deposition of interfacial layers and cathode electrode. According to our experimental requirements, the different thicknesses of interlayer materials were obtained separately by thermal evaporation on different ITO/PEDOT:PSS/P3HT:PCBM samples then followed by the deposition of a $100 \mathrm{~nm}$ thick top aluminum $(\mathrm{Al})$ electrode without breaking the vacuum of $\sim 1 \times 10^{-6}$ mbar. The Buckminster fullerene $\mathrm{C}_{60}$ and lithium fluoride ( $\mathrm{LiF}$ ) were used for thermal evaporation as received from Sigma-Aldrich. Bathocuproine (BCP) was purchased from LumTec and also used as received. The evaporation rate of the interfacial materials as well as of the Al was kept constant and as low as $1.0 \AA / \mathrm{s}$ in order to prevent the unwanted interpenetration into the active layer during thermal evaporation. The deposition rates of the various materials were calibrated by Dektak profilometer measurements then monitored by quartz microbalance during deposition. The active area of the devices was $0.2 \mathrm{~cm}^{2}$. Finally, the solar cell characterization was carried out in air using a $150 \mathrm{~W}$ Oriel Xenon-lamp solar simulator with an AM1.5G filter $\left(100 \mathrm{~mW} \mathrm{~cm}^{-2}\right)$. In all cases, the measurements were performed from a set of at least $16 \mathrm{PV}$ cells with each interlayer structure and the reported power conversion efficiency (PCE) values were taken as the average from those sets of measurements.

\section{Results}

In this study, we used several types of ultra-thin interlayers at the interface between the P3HT:PCBM photoactive layer and the cathode aluminum (Al) electrode to investigate their influence on polymer solar cell performance. Figure 1 illustrates the schematic diagram of a typical bulk heterojunction (BHJ) photovoltaic cell in which a nanoblend of P3HT:PCBM was used as a platform to conduct all the experiments with only changing type of interlayer materials and their respective thicknesses at the $\mathrm{BHJ} /$ cathode electrode interface.

First, we have probed bathocuproine (BCP) at the $\mathrm{BHJ} /$ cathode interface for the interfacial engineering of our polymer PV cells, since the BCP has been extensively studied for its exciton blocking nature in order to 
J.K. Baral et al.: Improved polymer solar cell performance by engineering of cathode interface

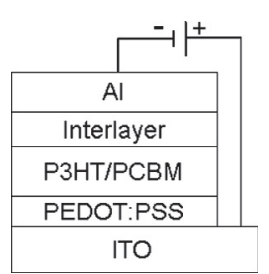

Fig. 1. The schematic illustration of the polymer photovoltaic cells investigated, namely, ITO/PEDOT:PSS/P3HT:PCBM/ Interlayer/Al.

Table 1. Key parameters obtained from the polymer solar cells of using varying thicknesses of $\mathrm{BCP}$ as an interfacial thin buffer layer compared to a pristine sample without any EBL at interface.

\begin{tabular}{ccccc}
\hline $\begin{array}{c}\text { BCP interlayer } \\
(\mathrm{nm})\end{array}$ & $\begin{array}{c}V_{o c} \\
(\mathrm{~V})\end{array}$ & $\begin{array}{c}J_{s c} \\
\left(\mathrm{~mA} / \mathrm{cm}^{2}\right)\end{array}$ & $\begin{array}{c}F F \\
(\%)\end{array}$ & $\begin{array}{c}\eta \\
(\%)\end{array}$ \\
\hline 0 & 0.50 & 3.15 & 53 & 0.83 \\
3 & 0.53 & 6.58 & 55 & 1.91 \\
6 & 0.53 & 7.45 & 61 & 2.40 \\
9 & 0.52 & 6.91 & 54 & 1.94 \\
\hline
\end{tabular}

improve the performance of the small molecule PV cells $[10,17-20,22]$. Figure 2a depicts the current density $(J)$ versus voltage $(V)$ measurement of the various BHJ solar cells fabricated by inserting a certain thickness of BCP between the P3HT:PCBM photoactive layer and the $\mathrm{Al}$ cathode. In Figure 2a, the dark solid spherical symbols $(\bullet)$ represent the $J-V$ characteristic of a control ITO/ PEDOT:PSS/P3HT:PCBM/Al solar cell made without any interfacial layer. It is plotted for comparison purpose with the other PV cells fabricated using interfacial layers. The control device without any interfacial layer showed fill factor $(\mathrm{FF})$ and power conversion efficiency (PCE) of $53 \%$ and $\sim 0.83 \%$ respectively. This low PCE value is expected for devices fabricated with no interlayer and ambient air exposure of the active layer before deposition of the cathode as was the case here. This pristine PV cell is only considered as a base reference for further comparison purpose, with other PV cells being engineered at their respective cathode interfaces. The power conversion efficiency (PCE) of the devices with varying thicknesses of BCP as buffer layer increases for a narrowly varying thickness $(3-6 \mathrm{~nm})$ of BCP interlayer as shown in Figure 2b. However, after merely increasing the thickness of BCP from $6 \mathrm{~nm}$ to $9 \mathrm{~nm}$, the device exhibits a decrease in the measured efficiency down to $1.94 \%$. Device performance improvement can be mostly associated with increasing $J_{s c}$ as observed in Figure $2 \mathrm{a}$ and the fill factor (FF) is also increased up to $61 \%$. However, no significant improvement occurred in open circuit voltage $V_{o c}$. The summarized key parameters are listed in Table 1 . The best performance, with $J_{s c}=7.45 \mathrm{~mA} / \mathrm{cm}^{2}, V_{o c}=0.53 \mathrm{~V}$ and $P C E=2.40 \%$ has been achieved with a $6 \mathrm{~nm}$ thick BCP layer at the Al interface.

The second material we have probed as an interlayer was lithium fluoride ( $\mathrm{LiF})$, which is one of the most widely studied interfacial materials in the field of organic

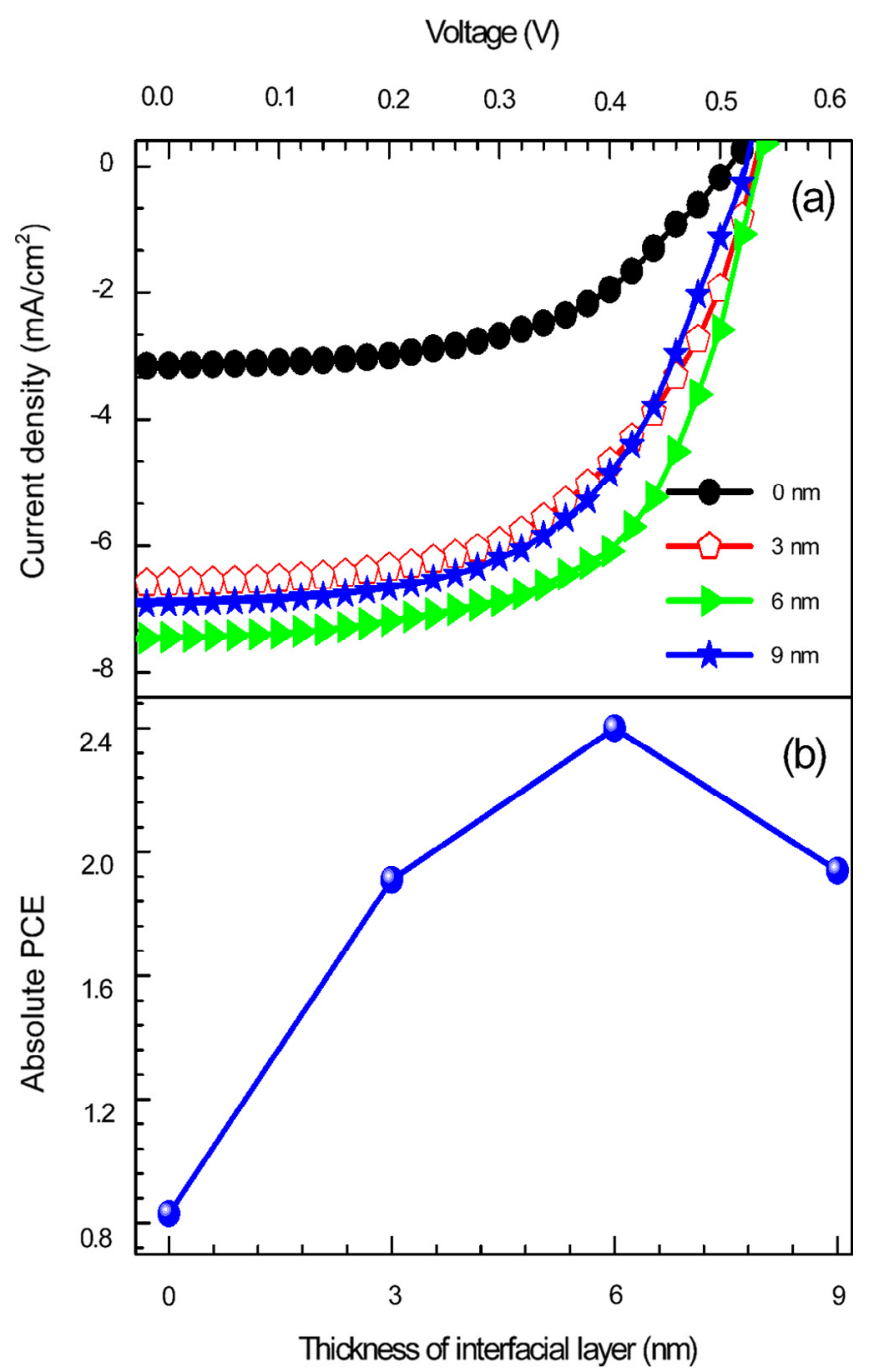

Fig. 2. (Color online) (a) The illuminated $J-V$ characteristics are measured under $100 \mathrm{~mW} \mathrm{~cm}^{-2}$ simulated AM1.5G spectrum, of the photovoltaic cells fabricated with varying thicknesses of thin buffer layers of BCP as an EBL at the interface. (b) Influence of varying thicknesses of $\mathrm{BCP}$ as a buffer layer on the device efficiency: the absolute PCE is plotted against the varying thicknesses of the BCP interfacial layer.

electronics, particularly in OLED and OPV research [9,23-25]. Figure 3a represents the current density as a function of voltage of several BHJ solar cells with varying thicknesses of $\mathrm{LiF}$ at the photoactive layer/cathode interface. The curve for a pristine control device without any $\mathrm{LiF}$ at the interface is also shown for comparison purpose. The key parameters from the devices based on LiF buffer layer at the interface of the solar cells are summarized in Table 2. From Figure 3a, it can be observed that there is a clear trend in significant increase of $J_{s c}$ with the introduction of a certain thickness LiF at the interface, which results in an effectively enhanced PCE. However, by slightly increasing further the thickness of LiF from $0.9 \mathrm{~nm}$ to $1.2 \mathrm{~nm}, J_{s c}$ decreases to a lower value, which results in a low performing device. The best performance, with $J_{s c}=11.02 \mathrm{~mA} / \mathrm{cm}^{2}, V_{o c}=0.54 \mathrm{~V}$ and 


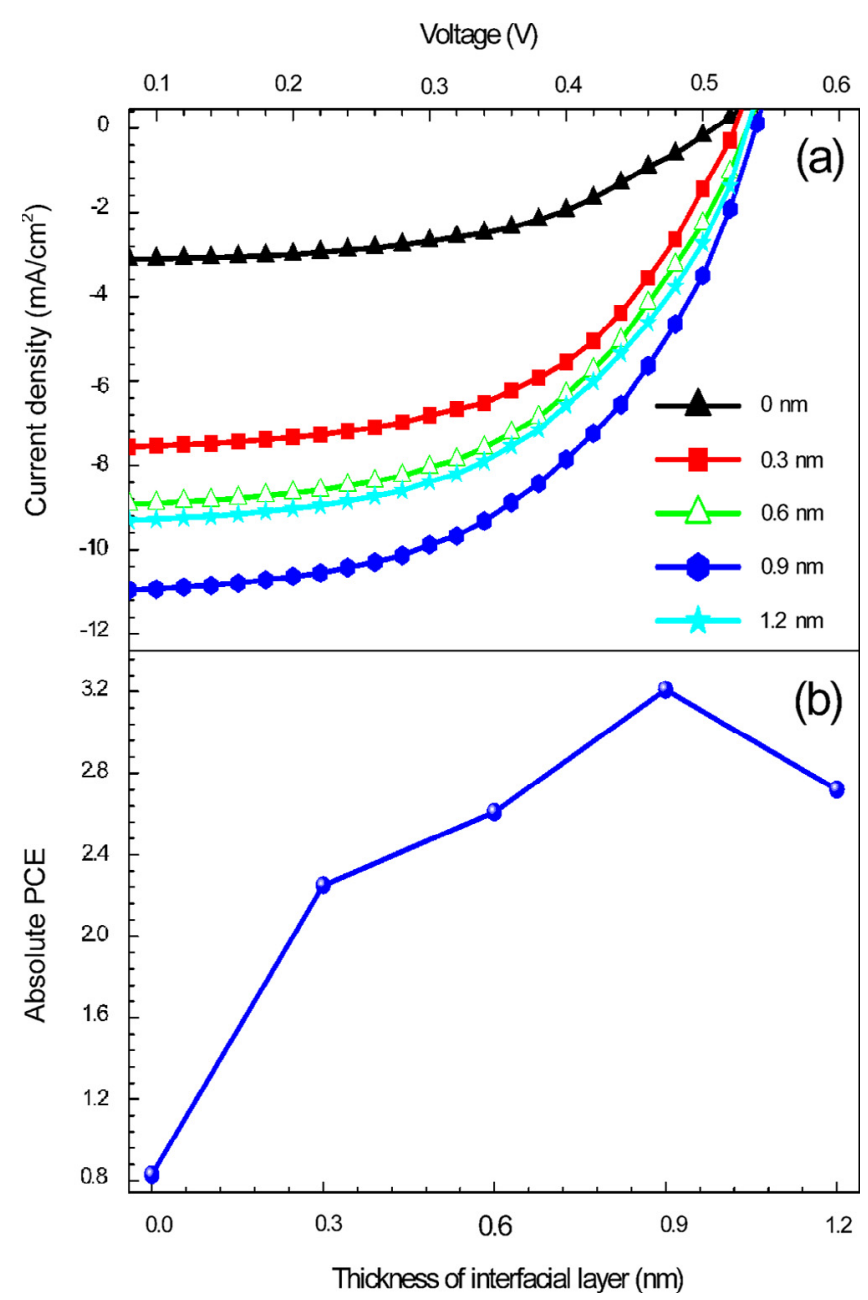

Fig. 3. (Color online) (a) The illuminated $J-V$ characteristics are measured under $100 \mathrm{~mW} \mathrm{\textrm {cm } ^ { - 2 }}$ simulated AM1.5G spectrum, of the PV cells fabricated with varying thicknesses of ultra-thin buffer layers of $\mathrm{LiF}$ as an EBL at the interface. (b) Influence of varying thicknesses of $\mathrm{LiF}$ as a buffer layer on the device efficiency: the absolute PCE is plotted as a function of varying thickness of $\mathrm{LiF}$ interfacial layer.

$P C E=3.21 \%$, has been achieved with a $0.9 \mathrm{~nm} \mathrm{LiF}$ interlayer. Figure $3 \mathrm{~b}$ and Table 2 also depict the increase in PCE that is merely due to the increase of $J_{s c}$ upon insertion of different thicknesses of $\mathrm{LiF}$ at the interface as it was the case with BCP interlayer-based devices.

Additionally, we investigated the use of an interlayer of a Buckminster fullerene $\mathrm{C}_{60}$ at the cathode. In Figure $4 \mathrm{a}$, the $J-V$ curves as a function of thickness of $\mathrm{C}_{60}$ are shown as well as the characteristics for a pristine device without any $\mathrm{C}_{60}$ interlayer. The $J-V$ curves clearly indicate that $J_{s c}$ significantly increases for the devices with a $\mathrm{C}_{60}$ interlayer in comparison to a device without any $\mathrm{C}_{60}$ interlayer and the efficiency increased following the increase in $J_{s c}$. The key parameters are summarized in Table 3. As the thickness of the $\mathrm{C}_{60}$ interlayer was raised from $3 \mathrm{~nm}$ to $12 \mathrm{~nm}$, the PCE increased more than twofold (from $0.83 \%$ to $1.92 \%$ ) as compared to the control pristine device without any $\mathrm{C}_{60}$ interlayer as shown in Figure $4 \mathrm{~b}$. However,
Table 2. Key parameters obtained from the polymer photovoltaic cells of using different thicknesses of $\mathrm{LiF}$ as an interfacial ultra-thin buffer layer compared to a virgin sample without any EBL at interface.

\begin{tabular}{ccccc}
\hline $\begin{array}{c}\text { LiF interlayer } \\
(\mathrm{nm})\end{array}$ & $\begin{array}{c}V_{o c} \\
(\mathrm{~V})\end{array}$ & $\begin{array}{c}J_{s c} \\
\left(\mathrm{~mA} / \mathrm{cm}^{2}\right)\end{array}$ & $\begin{array}{c}F F \\
(\%)\end{array}$ & $\begin{array}{c}\eta \\
(\%)\end{array}$ \\
\hline 0 & 0.50 & 3.15 & 53 & 0.83 \\
0.3 & 0.52 & 7.60 & 57 & 2.25 \\
0.6 & 0.53 & 8.97 & 55 & 2.61 \\
0.9 & 0.54 & 11.02 & 54 & 3.21 \\
1.2 & 0.53 & 9.35 & 55 & 2.72 \\
\hline
\end{tabular}

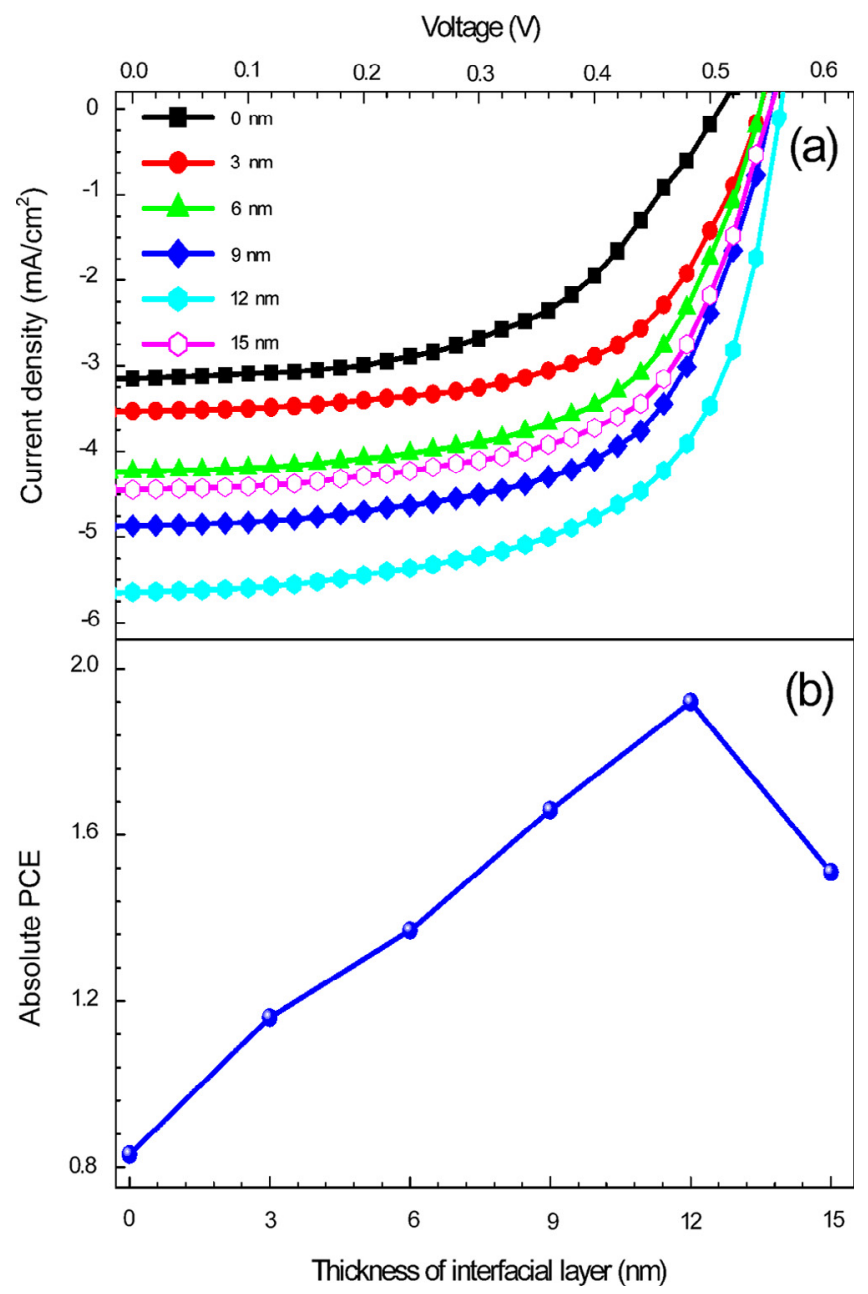

Fig. 4. (Color online) (a) The illuminated $J$ - $V$ characteristics

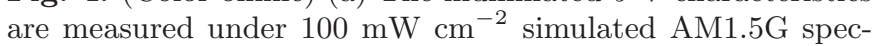
trum, of the solar cells fabricated with varying thicknesses of $\mathrm{C}_{60}$ at the interface. (b) Influence of varying thicknesses of $\mathrm{C}_{60}$ on the device efficiency: the absolute PCE is plotted as a function of varying thickness of $\mathrm{C}_{60}$ interlayer.

by increasing the $\mathrm{C}_{60}$ thickness from $12 \mathrm{~nm}$ to $15 \mathrm{~nm}$, $J_{s c}$ suddenly decreases to a lower value. The best performance with $J_{s c}=5.64 \mathrm{~mA} / \mathrm{cm}^{2}, V_{o c}=0.56 \mathrm{~V}, F F=61 \%$ and $P C E=1.92 \%$, has been achieved for a $12 \mathrm{~nm}$ thick $\mathrm{C}_{60}$ interlayer. 
Table 3. Key parameters obtained from the plastic PV cells with varying $\mathrm{C}_{60}$ interlayer thicknesses underneath $\mathrm{Al}$ electrode compared to a controlled device without any interlayer.

\begin{tabular}{ccccc}
\hline $\begin{array}{c}\mathrm{C}_{60} \text { overlayer } \\
(\mathrm{nm})\end{array}$ & $\begin{array}{c}V_{o c} \\
(\mathrm{~V})\end{array}$ & $\begin{array}{c}J_{s c} \\
\left(\mathrm{~mA} / \mathrm{cm}^{2}\right)\end{array}$ & $\begin{array}{c}F F \\
(\%)\end{array}$ & $\begin{array}{c}\eta \\
(\%)\end{array}$ \\
\hline 0 & 0.50 & 3.15 & 53 & 0.83 \\
3 & 0.54 & 3.53 & 61 & 1.16 \\
6 & 0.54 & 4.23 & 60 & 1.37 \\
9 & 0.55 & 4.87 & 62 & 1.66 \\
12 & 0.56 & 5.64 & 61 & 1.92 \\
15 & 0.55 & 4.44 & 62 & 1.51 \\
\hline
\end{tabular}

In the next experiment of interface engineering of BHJ photovoltaic cells, we have used a combination of BCP and $\mathrm{LiF}$ at the BHJ/cathode interface. From the previous experimental results of $\mathrm{BCP}$ - and LiF-based individual set of devices, the optimal thicknesses of BCP $(6 \mathrm{~nm})$ and $\mathrm{LiF}$ $(0.9 \mathrm{~nm})$ were taken, then slight variations of those values were tested. The $J-V$ characteristics and the absolute PCE versus the total interlayer thicknesses used in those sets of devices are presented in Figures $5 \mathrm{a}$ and $5 \mathrm{~b}$ respectively. The summarized key parameters are enlisted in Table 4. It is observed that when the optimal thickness of BCP $(6 \mathrm{~nm})$ and $\operatorname{LiF}(0.9 \mathrm{~nm})$ was used at the BHJ/cathode interface, it gives the optimal efficiency of $2.97 \%$. However, the performance of the PV cells fabricated from the optimal thickness of BCP $(6 \mathrm{~nm})$ and $\mathrm{LiF}(0.9 \mathrm{~nm})$ is inferior to that of the optimized $0.9 \mathrm{~nm}$ thick LiF-only device.

To further improve the photovoltaic cell performances, we have finally investigated a set of PV cells made using a combination of $\mathrm{C}_{60}$ and $\mathrm{LiF}$ at the $\mathrm{BHJ} /$ cathode interface. In these sets of experiments, we have used the optimal thickness $(0.9 \mathrm{~nm})$ of $\mathrm{LiF}$ in combination with varying thicknesses of $\mathrm{C}_{60}$. Figure 6 a represents the $J-V$ curves of photovoltaic cells of varying thicknesses of $\mathrm{C}_{60}$ interlayer. From Figure 6a, a clear trend of increase in $J_{s c}$ is observed upon introduction of $\mathrm{C}_{60}$ over a certain thickness range from $3 \mathrm{~nm}$ to $9 \mathrm{~nm}$ at the interface of $(0.9 \mathrm{~nm})$ $\mathrm{LiF} / \mathrm{Al}$, which results in significant improvement of PCE. However, by increasing the thickness of $\mathrm{C}_{60}$ interlayer from $9 \mathrm{~nm}$ to $12 \mathrm{~nm}$, the $J_{s c}$ starts to decrease, which produces a low performing device as shown in Figure 6b. The observed key parameters as extracted from the $J$ - $V$ curves of Figure 6 are summarized in Table 5 . It is clearly shown that key parameters like $V_{o c}, J_{s c}$ and PCE have been effectively improved by introducing the $\mathrm{C}_{60} / \mathrm{LiF}$ at the interface. With the use of a $\mathrm{C}_{60} / \mathrm{LiF}$ interlayer, devices with the highest PCE (3.94\%) among all the sets of fabricated photovoltaic cells, and with $J_{s c}$ and $V_{o c}$ of $13.04 \mathrm{~mA} / \mathrm{cm}^{2}$ and $0.56 \mathrm{~V}$, respectively, were achieved.

\section{Discussion}

In the previous section, we have seen that there is a common trend of improvement of the PCE of PV cells with the addition of a certain thickness of an interlayer between the active BHJ layer and the $\mathrm{Al}$ cathode electrode.

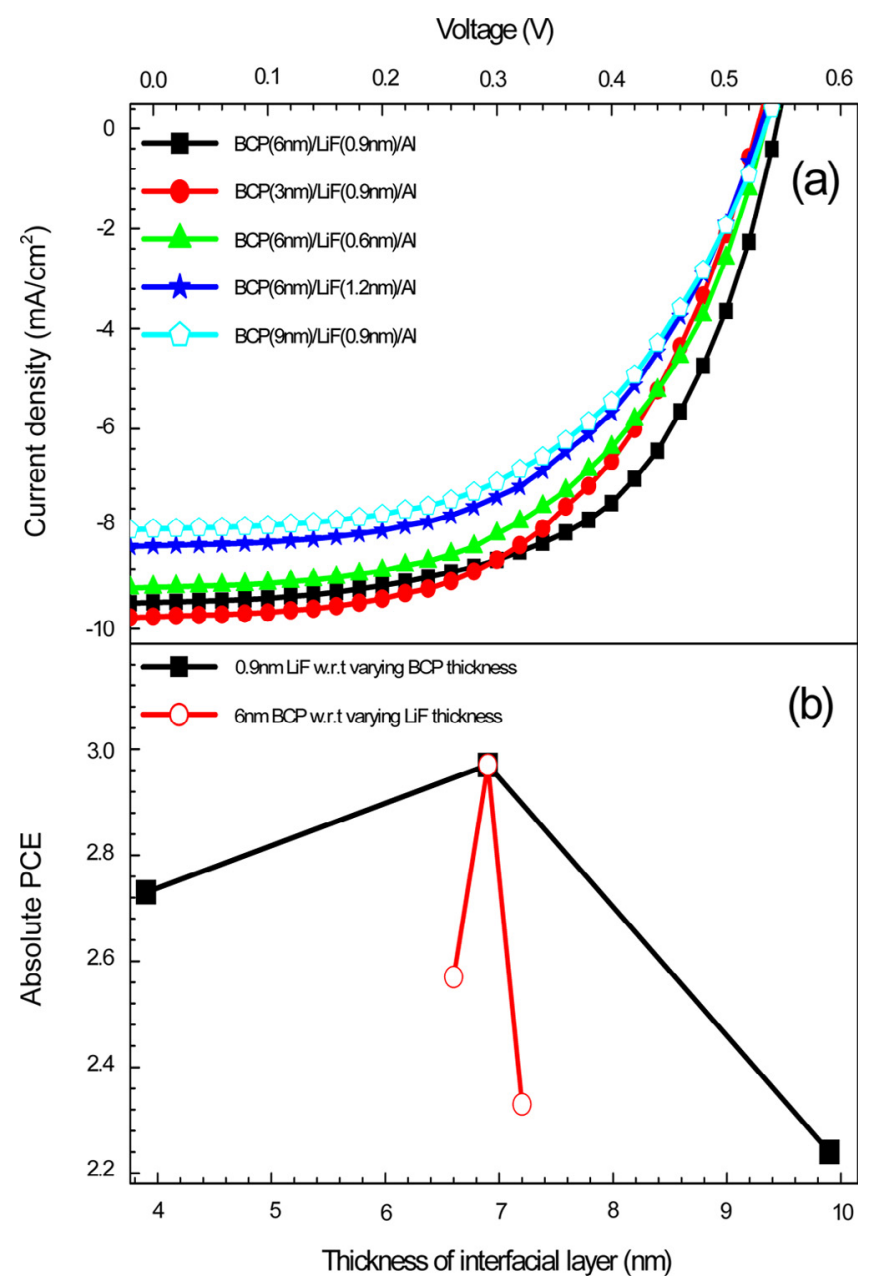

Fig. 5. (Color online) (a) The illuminated $J-V$ characteristics

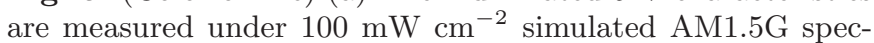
trum, of the solar cells fabricated with combination of varying thicknesses of $\mathrm{BCP} / \mathrm{LiF}$ at the interface. (b) Influence of combined buffer layer of $\mathrm{BCP} / \mathrm{LiF}$ on the device efficiency: the black solid squares ( $\mathbf{m}$ ) and hollow red spheres (O), respectively, represent the absolute PCE as a function of interlayer thickness for the devices fabricated with $0.9 \mathrm{~nm}$ thick LiF with varying $\mathrm{BCP}$ thicknesses and $6 \mathrm{~nm}$ thick $\mathrm{BCP}$ with varying $\mathrm{LiF}$ thicknesses.

Table 4. Key parameters obtained from the OPV cells fabricated with varying thicknesses of $\mathrm{BCP}$ with regard to $\mathrm{LiF}$ and vice versa, as interfacial buffer layers at the cathode interface.

\begin{tabular}{ccccc}
\hline $\begin{array}{c}\mathrm{BCP}(x \mathrm{~nm}) / \mathrm{LiF} \\
(y \mathrm{~nm})\end{array}$ & $\begin{array}{c}V_{o c} \\
(\mathrm{~V})\end{array}$ & $\begin{array}{c}J_{s c} \\
\left(\mathrm{~mA} / \mathrm{cm}^{2}\right)\end{array}$ & $\begin{array}{c}F F \\
(\%)\end{array}$ & $\begin{array}{c}\eta \\
(\%)\end{array}$ \\
\hline$(6) /(0.9)$ & 0.55 & 9.47 & 57 & 2.97 \\
$(3) /(0.9)$ & 0.53 & 9.75 & 53 & 2.73 \\
$(6) /(0.6)$ & 0.53 & 9.17 & 53 & 2.57 \\
$(6) /(1.2)$ & 0.53 & 8.33 & 53 & 2.33 \\
$(9) /(0.9)$ & 0.54 & 8.00 & 52 & 2.24 \\
\hline
\end{tabular}

However, the improvement of the performance is related to the nature of the interlayer materials used in our experiments. In this section, we will discuss the role of each interlayer material on the behavior of plastic photovoltaic cell performances. 


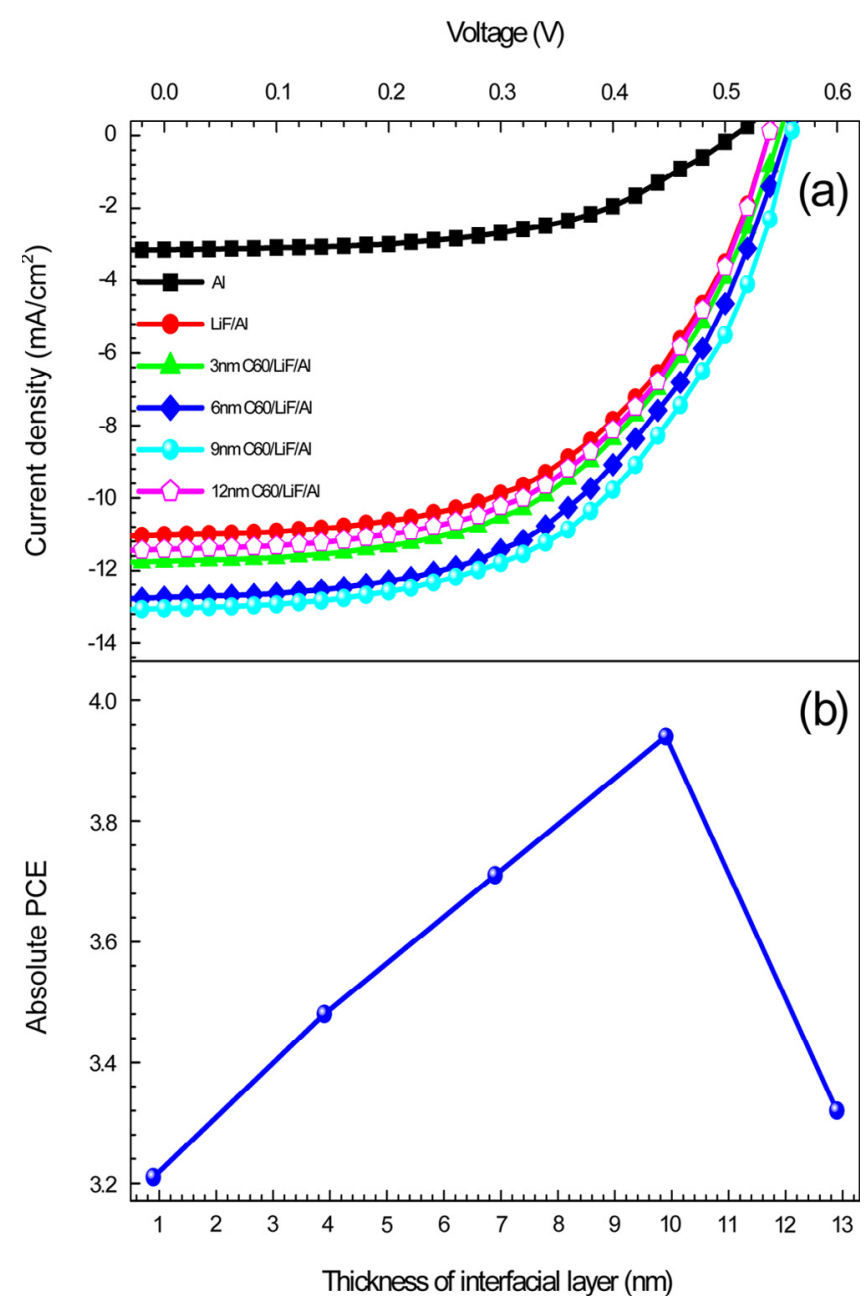

Fig. 6. (Color online) (a) The illuminated $J-V$ characteristics are measured under $100 \mathrm{~mW} \mathrm{~cm}^{-2}$ simulated AM1.5G spectrum, of the solar cells fabricated with combination of varying thicknesses of $\mathrm{C}_{60} / \mathrm{LiF}$ at the interface. (b) Influence of combined buffer layer of $\mathrm{C}_{60} / \mathrm{LiF}$ on the device efficiency: the absolute $\mathrm{PCE}$ as a function of interlayer thickness for the devices fabricated with $0.9 \mathrm{~nm}$ thick $\mathrm{LiF}$ with varying $\mathrm{C}_{60}$.

Table 5. Key parameters summarized from the characterization of plastic solar cells fabricated from varying thicknesses of $\mathrm{C}_{60}$ as an over layer with regard to $0.9 \mathrm{~nm} \mathrm{LiF} / \mathrm{Al}$ at the cathode interface.

\begin{tabular}{ccccc}
\hline $\begin{array}{c}\mathrm{C}_{60} \text { overlayer } \\
(\mathrm{nm})\end{array}$ & $\begin{array}{c}V_{o c} \\
(\mathrm{~V})\end{array}$ & $\begin{array}{c}J_{s c} \\
\left(\mathrm{~mA} / \mathrm{cm}^{2}\right)\end{array}$ & $\begin{array}{c}F F \\
(\%)\end{array}$ & $\begin{array}{c}\eta \\
(\%)\end{array}$ \\
\hline 0 & 0.54 & 11.02 & 54 & 3.21 \\
3 & 0.55 & 11.74 & 54 & 3.48 \\
6 & 0.55 & 12.74 & 53 & 3.71 \\
9 & 0.56 & 13.04 & 54 & 3.94 \\
12 & 0.54 & 11.41 & 54 & 3.32 \\
\hline
\end{tabular}

After photon absorption and exciton formation in organic solar cells, the exciton should reach a dissociation site, usually a donor/acceptor interface, and the separated charges then have to be transferred to the respective electrodes. If an exciton reaches an electrode before undergoing charge dissociation, it will be quenched and will not contribute to the photovoltaic effects. The low power conversion efficiency $(\sim 0.83 \%)$ of the reference P3HT:PCBM cell without any interlayer can then be attributed to various reasons, such as the poor Ohmic contact between the acceptor (PCBM) and the cathode, modest career transport across the electrode interface due to high energy barrier for electron extraction and the exciton quenching effect at the cathode interface. Forrest and co-workers $[10,21,26]$ have shown that the introduction of a thin large band gap organic material allows improving significantly the device performance of small molecule solar cells. They called it an "exciton blocking layer" (EBL) thin film, because its band gap is substantially larger than that of the organic donor and acceptor, which block excitons in the semiconducting layer far from the cathode avoiding any possible quenching effect at the organic/cathode interface $[10,21,26]$. The results presented in Figure 2 show that this is also the case in polymer BHJ PV cells. When a thin layer of large band gap material such as BCP is introduced between the photoactive layer and the cathode, the efficiency of the polymer solar cell significantly increases. However, the efficiency decreases at a certain point upon which the thickness reaches a critical value.

The curves in Figure 7 represent the normalized power conversion efficiency as well as series resistance as a function of thickness of the BCP interlayer used at the $\mathrm{BHJ} /$ cathode interface. The normalized PCE was determined from the performance of the $J-V$ curves under illumination and the series resistance $\left(R_{S}\right)$ was calculated from the slope of the $J-V$ characteristic curve in dark at $1 \mathrm{~V}$. It is clearly observed that the optimum normalized PCE directly corresponds to the lowest series resistance of the device. The reduction of the efficiency of the solar cell at a relatively thicker BCP layer is then due to an increase of the resistance of the entire cell due to the low conductivity of BCP. Hence, the BCP should be extremely thin in order not to restrict the electron extraction at the cathode [21].

Figure 8 shows the normalized PCE and the serial resistance as a function of $\mathrm{LiF}$ interlayer thickness. The efficiency increases with the increase in thickness of the interlayer up to a certain critical value then is reduced and the series resistance follows the same trend as it was the case for the BCP interlayer-based PV cells. However, the optimal thickness to achieve the high PCE is much lower than it was the case for $\mathrm{BCP}$ and the absolute $\mathrm{PCE}$ is also higher in this case. Moreover, the thickness needed to achieve the optimal PCE is low enough, which can barely make a continuous interlayer film. As the LiF possesses insulating properties, the thickness of such materials has to be extremely thin in order not to restrict the electron extraction to the cathode [27]. By merely increasing the thickness (from $0.9 \mathrm{~nm}$ to $1.2 \mathrm{~nm}$ ) of LiF at the interface, it hinders the electron transport across the $\mathrm{BHJ} /$ cathode interface, which results in a decrease in $J_{s c}$ and PCE as observed in these sets of devices. 
J.K. Baral et al.: Improved polymer solar cell performance by engineering of cathode interface

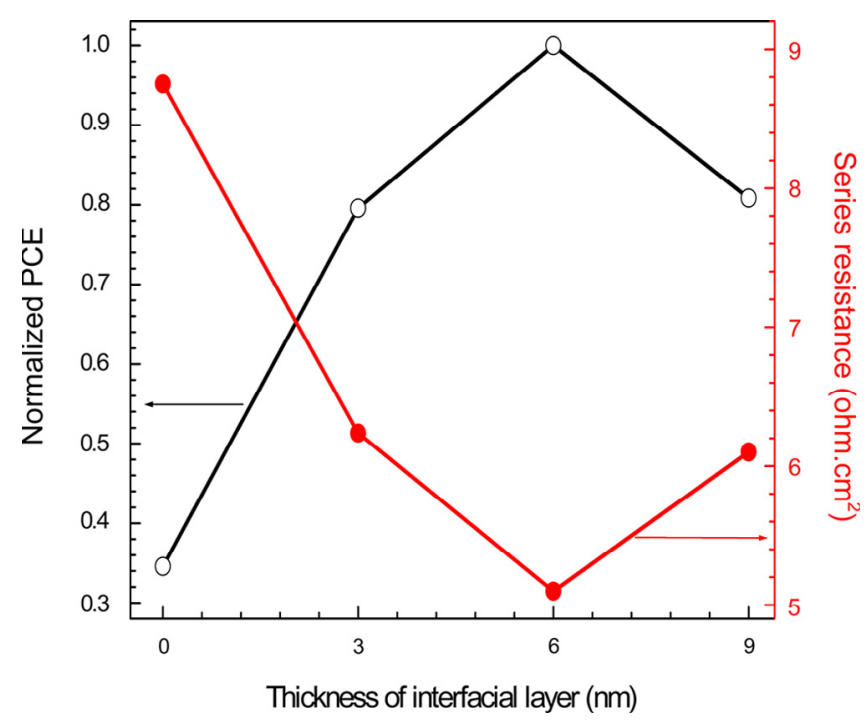

Fig. 7. (Color online) Influence of varying thicknesses of BCP as a buffer layer on device performance: the black hollow spheres (०) and red solid spherical $(\bullet)$ legends in the plot represent the normalized PCE and series resistance as a function of varying thickness of BCP interfacial layer.

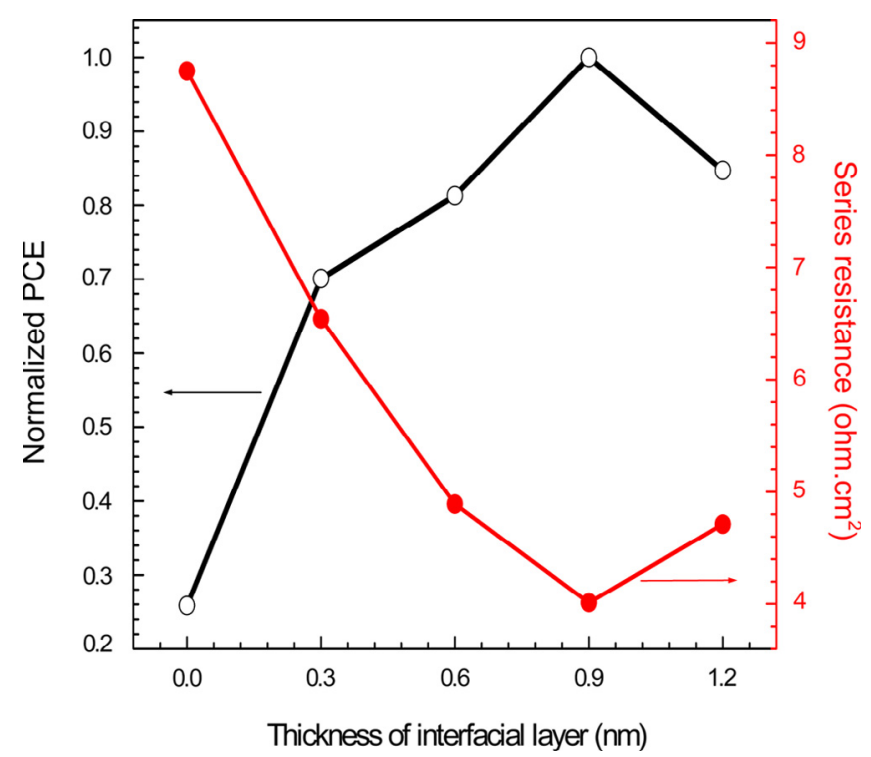

Fig. 8. (Color online) Influence of varying thicknesses of $\mathrm{LiF}$ as a buffer layer on device performance: the black hollow spheres $(\bigcirc)$ and red solid spherical $(\bullet)$ legends in the plot represent the normalized PCE and series resistance as a function of varying thickness of $\mathrm{LiF}$ interfacial layer.

Figure 9 shows a similar type of relationship between PCE and series resistance as a function of interlayer thickness for $\mathrm{C}_{60}$. In this case also, the highest efficiency corresponds to the lowest series resistance of the PV cells [28]. However, in this case, the range of the thickness is different from the previously observed BCP- and LiF-based devices. The $J_{s c}$ of the devices increased as a function of thickness of $\mathrm{C}_{60}$ overlayers up to $9 \mathrm{~nm}$. However, at a

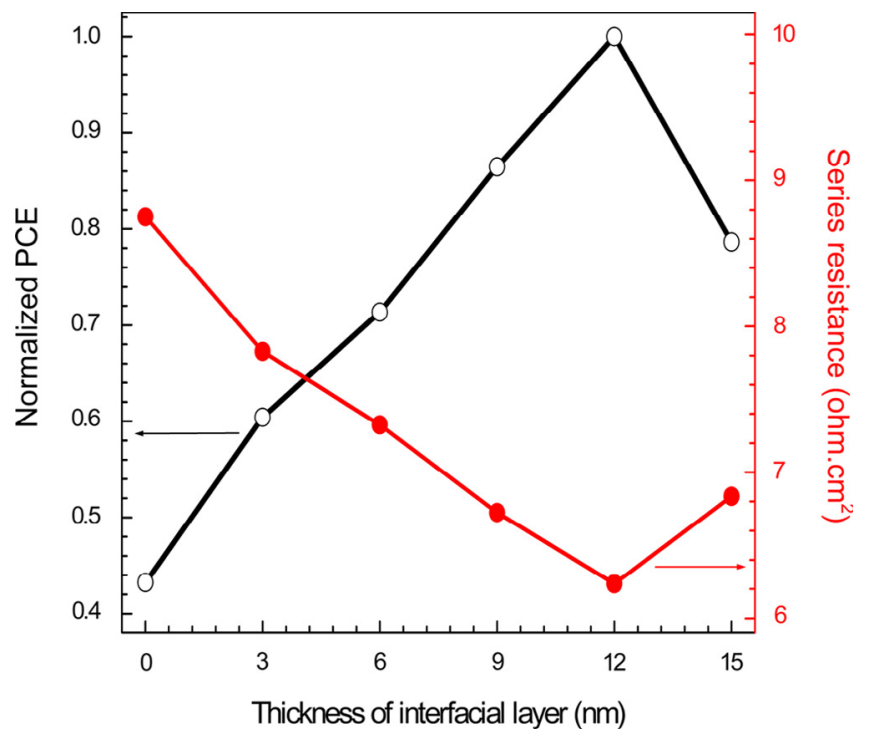

Fig. 9. (Color online) Influence of varying thicknesses of $\mathrm{C}_{60}$ as a buffer layer on device performance: the black hollow spheres $(\bigcirc)$ and red solid spherical $(\bullet)$ legends in the plot represent the normalized PCE and series resistance as a function of varying thickness of $\mathrm{C}_{60}$ interfacial layer.

certain thickness of $12 \mathrm{~nm}$ of $\mathrm{C}_{60}$ overlayer, the $J_{s c}$ started to decrease to a lower value.

In the pristine PV cells, the poor contact between the PCBM components of the active layer and the cathode electrode could also hinder the electron extraction from the active layer. This poor contact can be attributed to the unwanted vertical phase segregation in the BHJ nanoblend photoactive layer. This undesired vertical phase segregation usually occurred due to the solvent evaporation kinetics which causes the PCBM nanoparticles to segregate toward the bottom of the nanocomposite film to different extents depending upon the subtle variations in the BHJ film processing conditions $[11,29]$. The thermal evaporation of fullerenes introduces the additional fullerenes serving as electron transporting layer onto the top surface of the BHJ photoactive layer prior to the evaporation of cathode $\mathrm{Al}$ electrode reversing the unwanted vertical phase segregation [11]. Interposing $\mathrm{C}_{60}$ at the interface could also repair the poor contact at the electron acceptor/cathode interface, which builds an Ohmic contact and improves the charge extraction from the BHJ $[8,11]$ to the electrode. Additionally, interposing excess fullerenes at the cathode interface may prevent possible chemical reactions between P3HT and the Al cathode, which causes the poor device performance [11].

In order to compare the role of each individual interlayers at the interface, we have drawn a schematic illustration of the energy band scheme of the various structures used as interlayers, depicted in Figure 10. The schematic illustration for the energy-level diagram of the various materials and electrodes used in the experiments, presented with their work function, HOMO and LUMO values in electron volt $(\mathrm{eV})$ were taken from the references elsewhere $[22,30-33]$. In this figure, it is shown that 


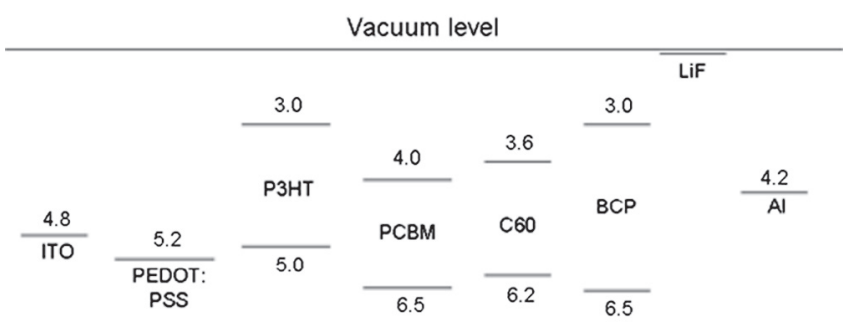

Fig. 10. The schematic illustration for the energy-level diagram of the various materials and electrodes used in the experiments, presented with their work function, HOMO and LUMO values in electron volt $(\mathrm{eV})$ were taken from the references elsewhere $[22,30-33]$.

the BCP has a larger band gap than that of PCBM, which efficiently blocks the excitons in order to prevent the undesired recombination, further decay and possible quenching $[10,21,26]$ at the metal electrode. As a result, in comparison to the pristine cells, these BCP interlayer-based devices exhibit an improved efficiency. For the case of $\mathrm{LiF}$ interlayer, there is no such exciton blocking effect expected from the energy band diagram as the energy level of LiF is almost considered to be at vacuum level. In this case, we presume that LiF could possibly lead to the creation of a dipole layer leading to a lowering of the energy barrier at the photoactive layer/cathode interface $[9,25]$. That could possibly contribute to the more efficient charge extraction across the interface and explains the enhancement of efficiency in our $\mathrm{LiF}$ interlayer-based devices. In the case of $\mathrm{C}_{60}$, the energy gap is not large enough to act as an efficient exciton blocking layer as was the case for BCP. In this case, introducing the $\mathrm{C}_{60}$ at the interface builds an Ohmic contact between electron transporting layer of PCBM components with cathode for the further improvement of excellent charge extraction $[8,11]$.

As the mechanism for PCE enhancement is different for the three explored materials combined interlayers could further enhance PCE. As it is shown earlier in Figure 5 that the varying thicknesses of both $\mathrm{BCP}$ in combination with $\mathrm{LiF}$ at the $\mathrm{BHJ} /$ cathode interfaces enhanced the PV cell performance as compared to the pristine cell, but those performances are inferior to that of the cells made with only LiF interlayer. Figure 11 shows that the efficiency is also directly related to the series resistance of devices and the resistance of the $\mathrm{BCP} / \mathrm{LiF}$ device is higher than that of the LiF-only device.

The normalized PCE and the serial resistance as a function of interlayer thickness for the $\mathrm{PV}$ cells made with varying thicknesses of $\mathrm{C}_{60}$ in combination with an optimized $0.9 \mathrm{~nm}$ thick of LiF are shown in Figure 12. Once more, the series resistance is lower for the highest efficiency cell. The most efficient PV cell was obtained with $(9 \mathrm{~nm}) \mathrm{C}_{60} /(0.9 \mathrm{~nm}) \mathrm{LiF}$ interlayer, having an efficiency of $3.94 \%$. Even though this efficiency (of $3.94 \%$ ) is lower than the efficiency reported from devices made with P3HT: PCBM blends where the complete process was performed under controlled atmosphere [34], this efficiency (of 3.94\%) compares well with reported PCE values from devices

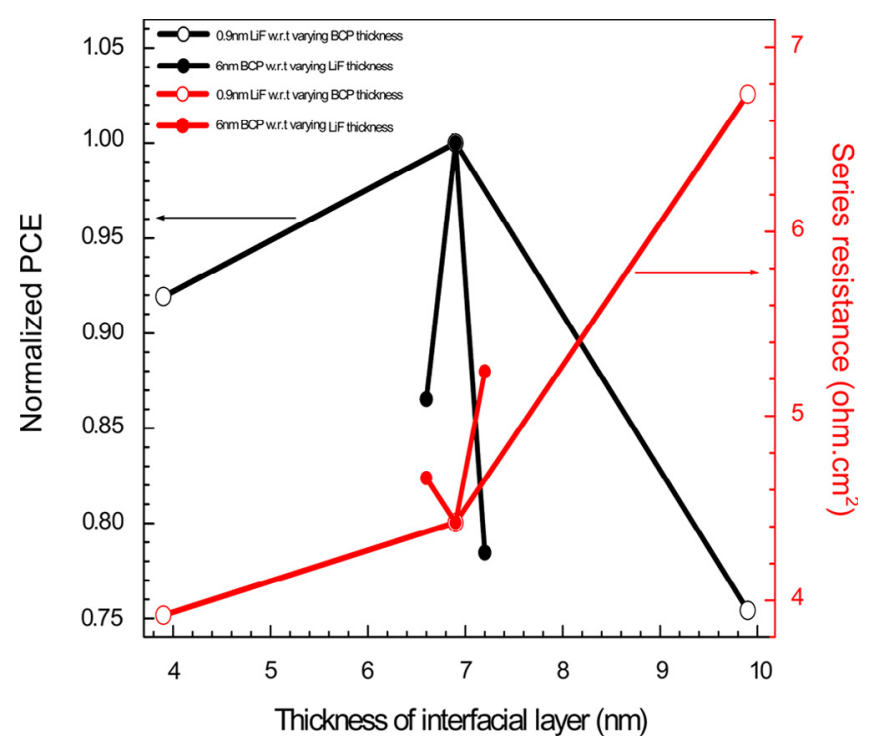

Fig. 11. (Color online) Influence of combination of varying thicknesses of $\mathrm{BCP} / \mathrm{LiF}$ as a buffer layer on device performance: the normalized PCE and series resistance curves are plotted as a function of varying thicknesses of $\mathrm{BCP}$ with regard to $0.9 \mathrm{~nm}$ thick $\mathrm{LiF}$ and varying thicknesses of $\mathrm{LiF}$ with regard to $6 \mathrm{~nm}$ thick BCP represented by the black and red hollow spheres $(\circ)$ and black and red solid spheres $(\bullet)$ respectively.

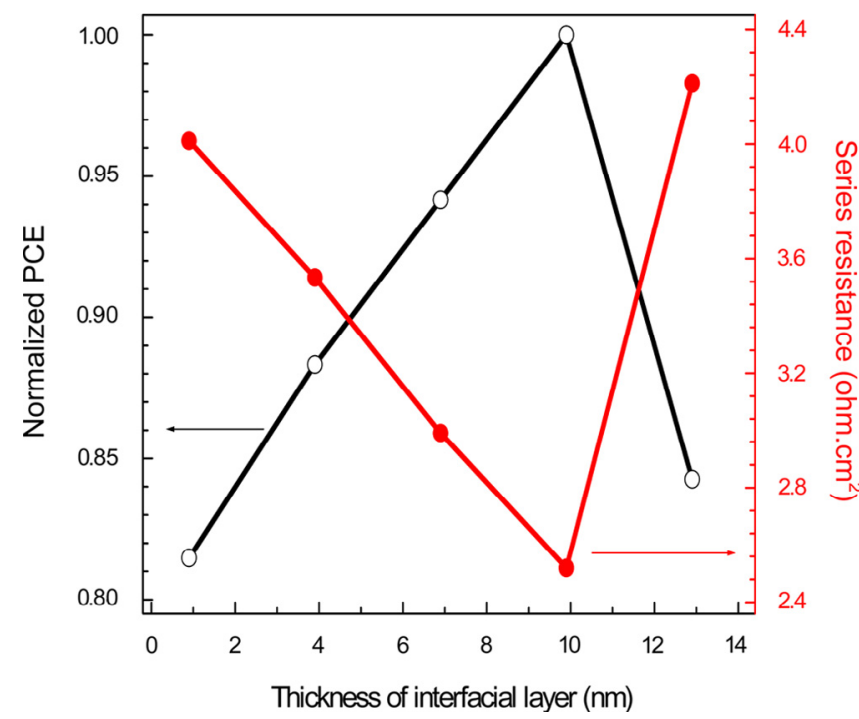

Fig. 12. (Color online) Influence of varying thicknesses of $\mathrm{C}_{60}$ with $0.9 \mathrm{~nm} \mathrm{LiF}$ as a buffer layer on device performance: the black hollow spheres $(\bigcirc)$ and red solid spherical $(\bullet)$ legends in the plot represent the normalized PCE and series resistance as a function of varying thickness of $\mathrm{C}_{60}$ with regard to a fixed $0.9 \mathrm{~nm}$ thick $\mathrm{LiF}$ at the interface.

fabricated through air exposure and is even higher than the recently reported PCE value of $3.54 \%$ for devices made with an interlayer of a $\mathrm{C}_{60}-\mathrm{LiF}$ mixture [13]. As the P3HT:PCBM active layer is extremely sensitive to photo-oxidation this effect was reported to be the primary degradation mechanism in OPVs [13]. Even the minimum 
J.K. Baral et al.: Improved polymer solar cell performance by engineering of cathode interface

amount of moisture and oxygen that might have diffused through the top layer in the first few hours of operation still managed to rapidly degrade the device. In another study, Dang et al. [35] observed that devices made by solution processing in air under ambient conditions systematically have lower power conversion efficiencies than devices made under controlled atmosphere conditions. We can then expect that the improvement of performance due to interface engineering will also be present on devices made under controlled atmosphere where no photo-oxidation of the active layer will take place. Further studies on this will be needed in order to observe this improvement.

Finally, Figure 13 shows the series resistance for the optimal cell of each interlayer as a function of optimal absolute PCE obtained. There is a clear and general trend observed, i.e., the photovoltaic cell performance in terms of optimal PCE increases with decrease in the series resistance across the PV cells and this improvement of the performance is independent of the nature of the interlayer used.

\section{Conclusions}

To summarize, the P3HT:PCBM BHJ PV cells were used as a platform to investigate the effects of inserting an ultra-thin buffer at the P3HT:PCBM active layer Al electrode interface. The control device has shown a PCE of $\sim 0.83 \%$. Interlayers of $\mathrm{BCP}, \mathrm{LiF}, \mathrm{C}_{60}$ and combination of $\mathrm{BCP}$ and $\mathrm{C}_{60}$ individually with $\mathrm{LiF}$ were studied. In all cases the PCE was increased as compared to the pristine device without any interlayer showing the importance of the role of the interlayer on the charge transfer at the active layer/electrode interface. For all the studied materials the PCE is inversely proportional to the series resistance of the devices independently of the nature of the interlayer. The optimal efficiency of $\sim 3.94 \%$ was obtained from the combination of $(9 \mathrm{~nm}) \mathrm{C}_{60}$ and $(0.9 \mathrm{~nm}) \mathrm{LiF}$ at the BHJ/cathode interface of the P3HT:PCBM PV cell. This efficiency represents an improvement of $23 \%$ compared to the standard device with LiF interlayer alone and is relatively high for devices fabricated and characterized with ambient air exposure. The highest efficiency can be attributed to the significantly improved charge collection and transport across the interface, which occurs upon introduction of the additional fullerenes between the active layer and the $\mathrm{LiF} / \mathrm{Al}$ cathode. The enhancement of the PCE obtained with the addition of interlayers shows the importance of electrode interface engineering in order to optimize charge transfer to maximize the power conversion efficiency in polymer solar cells. The present results should be applicable to other such similar systems in plastic photovoltaic cells.

We thank the Natural Science and Engineering Research Council of Canada (NSERC), the "Centre Québécois sur les Matériaux Fonctionnels" (CQMF) and the "Fonds Québécois de Recherche sur la Nature et les Technologies" (FQRNT) for the financial support to conduct this research work.

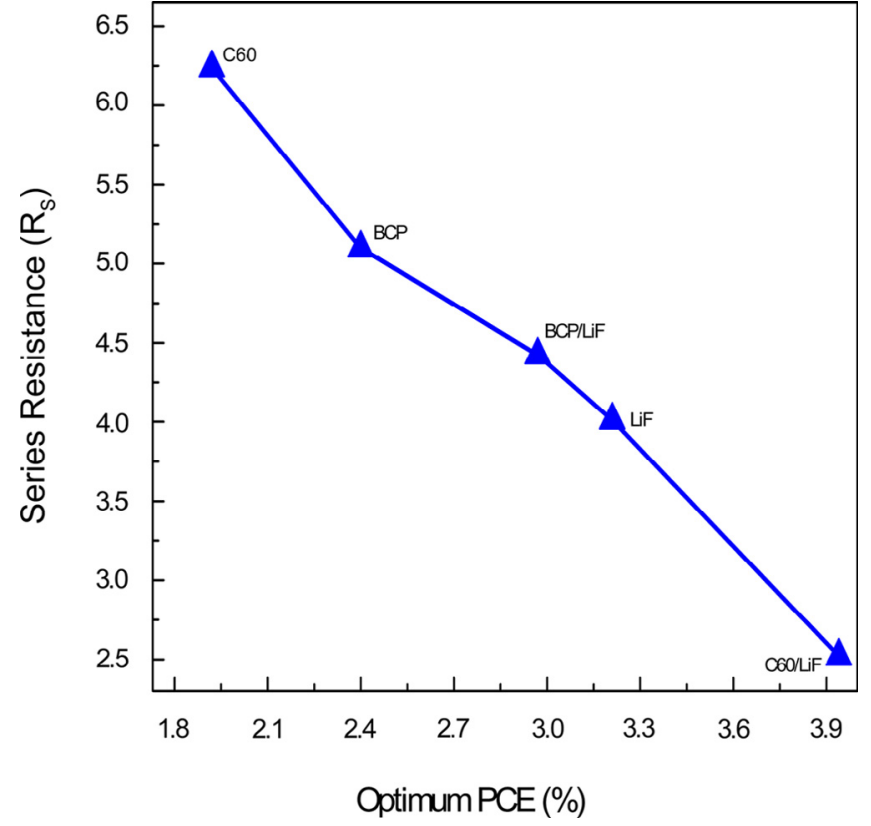

Fig. 13. (Color online) The series resistance (calculated from the slope of the $J-V$ characteristic curves in dark at $1 \mathrm{~V}$ of all PV cells) as a function of absolute value of maximum PCE ( $\eta)$, showing the increase in optimal PCE with decrease in series resistance of the cells using various interfacial layers of different thicknesses.

\section{References}

1. For general information about solar energy, consult the International Energy Agency Photovoltaic Power Systems Programme at www.iea-pvps.org (2010)

2. G. Li, V. Shrotriya, J. Huang, Y. Yao, T. Moriarty, K. Emery, Y. Yang, Nat. Mater. 4, 864 (2005)

3. J. Peet, A.J. Heeger, G.C. Bazan, Acc. Chem. Res. 42, $1700(2009)$

4. B. Kippelen, J.-L. Brédas, Energy Environ. Sci. 2, 251 (2009)

5. J. Peet, J.Y. Kim, N.E. Coates, W.L. Ma, D. Moses, A.J. Heeger, G.C. Bazan, Nat. Mater. 6, 497 (2007)

6. A.C. Mayer, S.R. Scully, B.E. Hardin, M.W. Rowell, M.D. McGehee, Mater. Today 10, 28 (2007)

7. A. Hadipour, B. de Boer, P.W.M. Blom, Adv. Funct. Mater. 18, 169 (2008)

8. L.-M. Chen, Z. Xu, Z. Hong, Y. Yang, J. Mater. Chem. 20, $2575(2010)$

9. C.J. Barabec, S.E. Shaheen, C. Winder, N.S. Sariciftci, Appl. Phys. Lett. 80, 1288 (2002)

10. P. Peumans, V. Bulovic, S.R. Forrest, Appl. Phys. Lett. 76, 2650 (2000)

11. B.T. de Villers, C.J. Tassone, S.H. Tolbert, B.J. Schwartz, J. Phys. Chem. C 113, 18978 (2009)

12. J.Y. Lee, Appl. Phys. Lett. 88, 073512 (2006)

13. D. Gao, M.G. Helander, Z.B. Wang, D.P. Puzzo, M.T. Greiner, Z.H. Lu, Adv. Mater. 22, 5404 (2010)

14. G. Dennler, M.C. Scharber, C.J. Brabec, Adv. Mater. 21, 1323 (2009)

15. C.J. Brabec, S. Gowrisanker, J.J.M. Halls, D. Laird, S. Jia, S.P. Williams, Adv. Mater. 22, 3839 (2010) 
16. S.-H. Lee, J.-H. Kim, T.-H. Shim, J.-G. Park, Electron. Mater. Lett. 5, 47 (2009)

17. Z.B. Wang, M.G. Helander, M.T. Greiner, Z.H. Lu, Appl. Phys. Lett. 95, 043302 (2009)

18. V. Choong, Y. Park, Y. Gao, T. Wehrmeister, K. Mullen, B.R. Hsieh, C.W. Tang, Appl. Phys. Lett. 69, 1492 (1996)

19. T. Dienel, H. Proehl, R. Forker, K. Leo, T. Fritz, J. Phys. Chem. C 112, 9056 (2008)

20. Z.R. Hong, Z.H. Huang, X.T. Zeng, Thin Solid Films 515, 3019 (2007)

21. B.P. Rand, J. Li, J.G. Xue, R.J. Holmes, M.E. Thompson, S.R. Forrest, Adv. Mater. 17, 2714 (2005)

22. A.S. Yapi, L. Toumi, Y. Lare, G.M. Soto, L. Cattin, K. Toubal, A. Djafri, M. Morsli, A. Khelil, M.A.D. Valle, J.-C. Bernède, Eur. Phys. J. Appl. Phys. 50, 30403 (2010)

23. L.S. Hung, C.W. Tang, M.G. Mason, Appl. Phys. Lett. 70, $152(1997)$

24. M. Matsumura, K. Furukawa, Y. Jinde, Thin Solid Films 331, 96 (1998)

25. M.O. Reese, M.S. White, G. Rumbles, D.S. Ginley, S.E. Shaheen, Appl. Phys. Lett. 92, 053307 (2008)
26. P. Peumans, S.R. Forrest, Appl. Phys. Lett. 79, 126 (2001)

27. B. Paci, A. Generosi, V.R. Albertini, P. Perfetti, R. de Bettignies, J. Leroy, M. Firon, C. Sentein, Appl. Phys. Lett. 89, 043507 (2006)

28. A. Kumar, G. Li, Z. Hong, Y. Yang, Nanotechnology 20, $165202(2009)$

29. M.C. Quiles, T. Ferenczi, T. Agostinelli, P.G. Etchegoin, Y. Kim, T.D. Anthopoulos, P.N. Stavrinou, D.D.C. Bradley, J. Nelson, Nat. Mater. 7, 158 (2008)

30. F.C. Krebs, J. Alstrup, H. Spangaard, K. Larsen, E. Kold, Sol. Energy Mater. Sol. Cells 83, 293 (2004)

31. J.Y. Lee, Mol. Cryst. Liq. Cryst. 499, 11/[333] (2009)

32. A.W. Hains, T.J. Marks, Appl. Phys. Lett. 92, 023504 (2008)

33. D.H. Wang, H.K. Lee, D.-G. Choi, J.H. Park, O. Park, Appl. Phys. Lett. 95, 043505 (2009)

34. W. Ma, C. Yang, X. Gong, K. Lee, A.J. Heeger, Adv. Funct. Mater. 15, 1617 (2005)

35. M.-T. Dang, G. Wantz, L. Hirsch, J.-P. Parneix, ECS Trans. 23, 449 (2009) 